\title{
Thermodynamics of a frustrated quantum magnet on a square lattice
}

\author{
K. Yu. Povarov, ${ }^{1,}$ 用 V. K. Bhartiya, ${ }^{1}$ Z. Yan, ${ }^{1}$ and A. Zheludev ${ }^{1}$ \\ ${ }^{1}$ Laboratory for Solid State Physics, ETH Zürich, 8093 Zürich, Switzerland
}

(Dated: January 14, 2019)

\begin{abstract}
We report the magnetic and calorimetric measurements in single crystal samples of the square lattice $J_{1}-J_{2}$ quantum antiferromagnet $\mathrm{BaCdVO}\left(\mathrm{PO}_{4}\right)_{2}$. An investigation of the scaling of magnetization reveals a "dimensionality reduction" indicative of a strong degree of geometric frustration. Below a characteristic temperature of $T^{*} \simeq 150 \mathrm{mK}$ we observe the emergence of an additional strongly fluctuating quantum phase close to full magnetic saturation. It is separated from the magnetically ordered state by first- and second-order phase transitions, depending on the orientation of the applied magnetic field. We suggest that this phase may indeed be related to the theoretically predicted spin-nematic state.
\end{abstract}

\section{INTRODUCTION}

The quest for the so-called spin-nematic state in magnetic insulators started more than three decades ago, but continues to this day [1] [ This exotic magnetic order spontaneously breaks rotational symmetry, while keeping time-reversal symmetry intact. It can be understood as a quantum condensate of bound magnon pairs [2 4]. The key characteristics of any potential host system are competing ferro- (FM) and antiferromagnetic (AF) interactions and extreme quantum fluctuations. The baseline model is the $S=1 / 2$ square lattice Heisenberg Hamiltonian with FM nearest-neighbor exchange $J_{1}$ and AF next-nearest-neighbor coupling $J_{2}$ [3, 7-10] sketched in Fig. 1(a) alongside its phase diagram. The classical critical point at $J_{2} / J_{1}=-1 / 2$ separates FM and columnarAF states, but becomes destabilized by quantum fluctuations and is replaced by a novel region in its vicinity. The resulting state can be understood as a magnon bound pair condensate occurring at zero field - the spinnematic [3, 9]. Even outside the narrow $J_{2} / J_{1}$ parameter range in which spin nematic is supposed to exist at zero field, this state can be further stabilized in a magnetized system. Magnon pair condensation and hence spin nematicity can be induced by an external magnetic field close to the saturation point. This result turns out to hold well away from optimal parameter set $J_{2} / J_{1}=-1 / 2$ and even in the presence of additional couplings in the Hamiltonian [3, 11.

Despite the vast body of theoretical work, experimentally the spin nematic state on a frustrated square lattice remains elusive. One obvious problem is that the tensorial order parameter is invisible to most conventional magnetism probes. What is an even bigger obstacle, is that potential model compounds are few and hard to synthesize. The most promising known candidate is $\mathrm{BaCdVO}\left(\mathrm{PO}_{4}\right)_{2}$ [12, 13. The applicability of the $J_{1}-J_{2}$ model to this compound has been validated by density functional theory calculations [14. The

\footnotetext{
* povarovk@phys.ethz.ch

$\dagger$ http://www.neutron.ethz.ch/
}
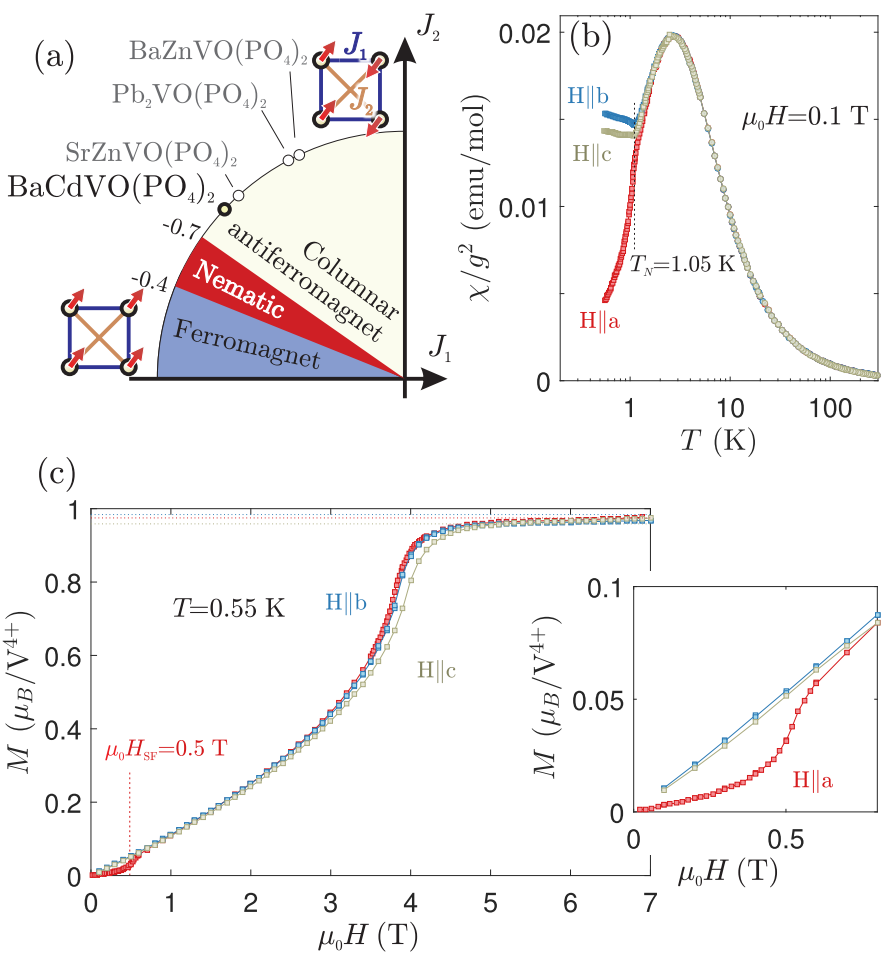

FIG. 1. (a) The frustrated $S=1 / 2$ Heisenberg square lattice model and typical "circular" representation of its ground state as a function of the ferromagnetic $J_{1}$ to antiferromagnetic $J_{2}$ exchange ratio. The positions of $\mathrm{BaCdVO}\left(\mathrm{PO}_{4}\right)_{2}$ as well as of few other similarly structured materials are shown (after Ref. [12]). (b) Magnetic susceptibilities along the different directions of $\mathrm{BaCdVO}\left(\mathrm{PO}_{4}\right)_{2}$ crystal, scaled with their $g$-factors. (c) Isothermal magnetization along different directions at $T=0.55 \mathrm{~K}$. The inset shows the region around $\mu_{0} H_{\mathrm{SF}} \simeq 0.5 \mathrm{~T}$ in more detail. The dashed lines show the estimate for $M_{\text {sat }}=g \mu_{B} / 2$ value. All the magnetic data is corrected for the diamagnetic background.

material features strong geometric frustration $\left(J_{2} / J_{1} \simeq\right.$ -0.9 ) and easily accessible energy scales (saturation field about $4 \mathrm{~T}$, AF order below $T_{N}=1.05 \mathrm{~K}$ ). The hightemperature thermodynamics is consistently described by $J_{1}=-3.6 \mathrm{~K}$ and $J_{2}=3.2 \mathrm{~K}[12]$. For lack of other candidates, $\mathrm{BaCdVO}\left(\mathrm{PO}_{4}\right)_{2}$ has been a subject of intense 


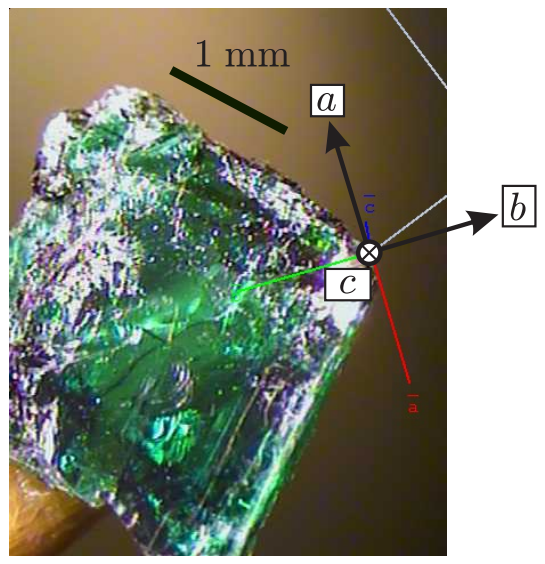

FIG. 2. A single 16-mg crystal sample of $\mathrm{BaCdVO}\left(\mathrm{PO}_{4}\right)_{2}$ used in magnetization measurements. A snapshot from a Bruker APEX II single-crystal x-ray diffractometer.

theoretical studies, including specific predictions for inelastic neutron scattering [15] and nuclear magnetic resonance 16. Disappointingly, a lack of single crystals has severely impeded experimental studies. To date, no empirical evidence of a spin-nematic phase or any related unconventional magnetism has been reported in this material.

In the present paper we describe the unusual magnetic and thermodynamic properties of single-crystal samples of $\mathrm{BaCdVO}\left(\mathrm{PO}_{4}\right)_{2}$. We map out the anisotropic magnetic phase diagram and study the "dimensionality reduction" and peculiar scaling of magnetization near the field-induced quantum phase transition. We accomplish this by employing magnetization, specific heat, and the magnetocaloric effect studies. In what may be the first sign of spin nematicity, we report evidence of an additional low-temperature field-induced strongly fluctuating quantum regime just below saturation. In an axially symmetric geometry the new state emerges in a firstorder transition, and is preceded by substantial precursor transverse fluctuations in the magnetically ordered state.

\section{EXPERIMENT DETAILS}

\section{A. Material}

High-quality single crystals of $\mathrm{BaCdVO}\left(\mathrm{PO}_{4}\right)_{2}$ were grown using the self-flux Bridgman method from the melt of presynthesized $\mathrm{BaCdP}_{2} \mathrm{O}_{7}$ and vanadium dioxide at $1000^{\circ} \mathrm{C}$. The details of the method will be published elsewhere. The crystal structure [orthorhombic $P_{b c a}\left(D_{2 h}^{15}\right.$, No 61$), a=8.84, b=8.92, c=19.37 \AA]$ was validated using single-crystal x-ray diffraction on a Bruker APEXII instrument, and found to be totally consistent with that reported previously [17.

The single crystals of $\mathrm{BaCdVO}\left(\mathrm{PO}_{4}\right)_{2}$ have the appearance of green transparent square plates. The plate corresponds to the crystallographic $a b$ plane, with the directions of $a$ and $b$ axes being usually along the diagonals. Correspondingly, the $c$ axis is normal to the plane. One of the crystals from the present study is shown in Fig. 2 .

We would like to note that the highly symmetric shape of the samples ensures that no crystal morphologyrelated effects can be expected to make a difference between $\mathbf{H} \| \mathbf{a}$ and $\mathbf{H} \| \mathbf{b}$ configurations.

\section{B. Techniques}

The magnetization measurements were performed with the $7 \mathrm{~T}$ SQUID magnetometer [Quantum Design Magnetic Property Measurement System (MPMS)] in the temperature range $1.8-300 \mathrm{~K}$. Further extension to the temperatures of about $0.5 \mathrm{~K}$ was achieved with the help of ${ }^{3}$ He cryostat inset iQuantum iHelium3. The magnetic susceptibility $\chi=M / H$ was measured at a small field $0.1 \mathrm{~T}$. The crystal shown in Fig. 2 was used in all the magnetic measurements.

Specific heat measurements were carried out on a standard Quantum Design relaxation calorimetry option and the ${ }^{3} \mathrm{He}-{ }^{4} \mathrm{He}$ dilution refrigerator inset for Quantum Design Physical Properties Measurement System (PPMS). Two measurement geometries $\mathbf{H} \| \mathbf{a}$ and $\mathbf{H} \| \mathbf{b}$ were realized by mounting a 2.3-mg flat single-crystal sample on a small silver foil holder with Apiezon $\mathrm{N}$ grease. The measurement procedure consists of giving a gentle heat pulse to the sample platform. Then the temperature rise is observed during the pulse, and subsequent temperature fall is observed as the heater is turned off. The resulting $T(t)$ curve typically has a characteristic "shark fin" shape, and specific heat can be calculated from the curvature.

The magnetocaloric effect measurements were performed in the same setup by directly reading the resistivity of the sample thermometer as the function of slowly varying magnetic field. It was done either with an $\mathrm{Ag}$ ilent E4980A LCR meter, or with a Stanford Research SR830 lock-in amplifier.

\section{RESULTS AND DISCUSSION}

\section{A. Magnetization studies \\ 1. Susceptibility above $T_{N}$}

Above $T_{N}$ the susceptibilities [Fig. 1(b)] show qualitatively identical behavior: typical Curie-Weiss tail at high temperatures, followed by a rounded maximum at $T \simeq$ $2.5 \mathrm{~K}$ and then gradual decrease down to $T_{N}=1.05 \mathrm{~K}$ marked by a kink.

The Curie-Weiss part of the susceptibility curve at high temperatures can be used for accurate determination of the $g$ factors and the diamagnetic background: 


\begin{tabular}{ccc}
\hline \hline Direction & $g_{\alpha}$ & $\chi_{\alpha}^{0}(\mathrm{emu} / \mathrm{mol})$ \\
\hline $\mathbf{a}$ & $1.95(1)$ & $-1.79(2) \times 10^{-4}$ \\
$\mathbf{b}$ & $1.97(1)$ & $-2.45(2) \times 10^{-4}$ \\
$\mathbf{c}$ & $1.92(1)$ & $-2.32(2) \times 10^{-4}$ \\
\hline \hline
\end{tabular}

TABLE I. Results of the Curie-Weiss analysis of the hightemperature susceptibility.

$$
\chi_{\alpha}(T)=\chi_{\alpha}^{0}+\frac{\left(g_{\alpha} / 2\right)^{2} C}{T+\Theta},
$$

where $C=0.375 \mathrm{~K} \mathrm{emu} / \mathrm{mol}$ is the Curie constant for the $S=1 / 2$ case with $g=2.00$. The analysis given by Eq. (1) was performed in a temperature window between 30 and $200 \mathrm{~K}$. The Curie-Weiss temperature is found to be $\Theta=-0.90(2) \mathrm{K}$; we have enforced the equal value for all three directions. The values of $g$ factors and diamagnetic background susceptibilities $\chi_{\alpha}^{0}$ are summarized in Table $\mathbb{I}$. The obtained $g$-factor values are rather isotropic and consistent with powder EPR estimates [18]. In Fig. 1(b) one can see that background subtracted susceptibilities normalized by $g^{2}$ show a perfect overlap in absence of magnetic order.

\section{Easy-axis anisotropy}

Below the Néel temperature $T_{N}=1.05 \mathrm{~K}$ the susceptibilities shown in Fig. 1(b) start to show rather different behavior. At low temperatures $\chi_{b}(T)$ and $\chi_{c}(T)$ remain more or less constant, while $\chi_{a}(T)$ shows a rapid decrease upon cooling. This suggests a collinear magnetic structure with spins along the a axis. This interpretation is backed by isothermal magnetization $M(H)$ scans at $T=0.55 \mathrm{~K}$ [Fig. 1(c)]. For the $\mathbf{H} \| \mathbf{a}$ case (and only for that geometry) there is a pronounced magnetization jump around $\mu_{0} H_{\mathrm{SF}} \simeq 0.5 \mathrm{~T}$. This behavior is characteristic of a spin-flop transition driven by the weak Ising-like anisotropy, a being the magnetic easy axis. Thus, using a simple Heisenberg model to describe the system can only be done with caution. Below we shall refer to experiments with $\mathbf{H} \| \mathbf{a}$ as the axial geometry, and to those with a field in perpendicular directions as transverse.

\section{Convex shape and "dimensionality reduction"}

The most striking feature of the measured $M(H)$ curves is their extreme convex shape close to the saturation. As known from the numerical studies of the $J_{1}-J_{2}$ model [13, 19, it serves as a reliable indicator of the significant magnetic frustration, indirectly confirming the nearly critical positioning of $\mathrm{BaCdVO}\left(\mathrm{PO}_{4}\right)_{2}$ on the Fig. 1(b) phase diagram.

We note that the measured convex magnetization curve is reminiscent of the cusp singularity occurring
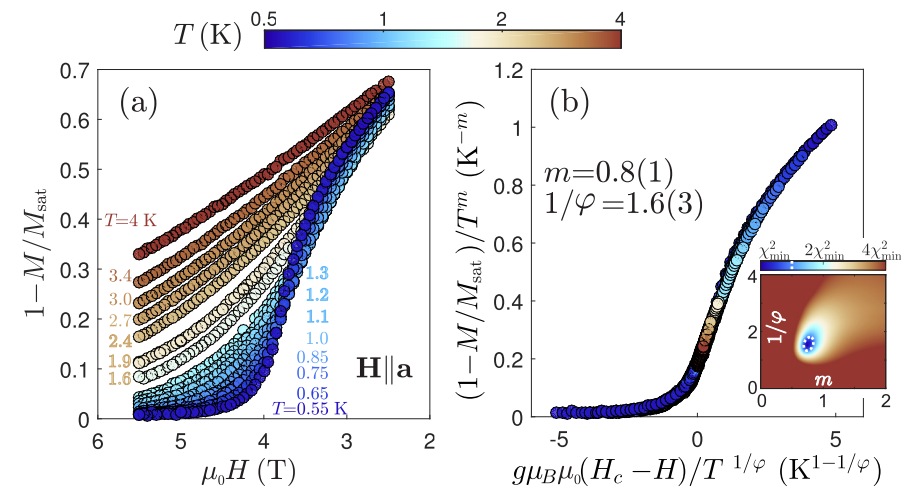

FIG. 3. Scaling of magnetization, observed near the saturation field ( $\mathbf{H} \|$ a case). (a) Raw data, taken in the interval $0.5-4$ K. (b) Same data, scaled according to Eq. (4). The observed exponents are $1 / \varphi=1.6(3)$ and $m=0.8(1)$. The inset shows the empirical $\chi^{2}$ goodness of overlap with highlighted boundary at which the optimal value increases by $50 \%$.

at saturation in the AF spin chains [20, 21]. This feature - square root cusp at the saturation magnetization $M_{\text {sat }}-M(H) \propto \sqrt{H_{c}-H}$ - is endemic to one dimension, yet it appears in our essentially two-dimensional (2D) material. This is another signature of the frustration, known as the "dimensionality reduction". For example, a similar effect is responsible for low-temperature crossover to effectively $2 \mathrm{D}$ behavior in a nominally $3 \mathrm{D}$ material $\mathrm{BaCuSi}_{2} \mathrm{O}_{6}$ ("Han purple") [22. In the case of the frustrated square lattice a qualitative prediction is given by Jackeli and Zhitomirsky [23]: close to the points of perfect frustration $\left|J_{2} / J_{1}\right|=1 / 21 \mathrm{D}$-like behavior with a square root magnetization cusp is indeed present in a 2D material at saturation. A simple explanation is, close to $H_{c}$ the low-energy part of the spin-wave spectrum defining the low- $T$ behavior features a continuous circle of degenerate minima as the result of strong frustration. This effectively reduced the problem to a one-dimensional one, rendering the low-energy spectrum as being pseudo-1D 23].

Thus, verifying the zero temperature $M_{\text {sat }}-M(H) \propto$ $\sqrt{H_{c}-H}$ prediction would be a strong signature of nearly critical $J_{2} / J_{1}$ coupling ratio in $\mathrm{BaCdVO}\left(\mathrm{PO}_{4}\right)_{2}$. However, in a realistic experiment we are dealing with the finite temperatures that make the cusp rounded and hide away the associated power law. Below we will show that by considering the quantum critical behavior of longitudinal magnetization close to $H_{c}$ it is nonetheless possible to relate the "hidden" zero temperature cusp to the available finite temperature data.

\section{Quantum critical scaling: Theory}

The basic assumption that we need to make is that the hyperscaling holds at the quantum critical point. This would be the case if the "dimensionality reduction" scenario takes place indeed. Then in the vicinity of the 
transition the free energy can be expressed as

$$
F(T, H)=\lambda^{b} \mathcal{F}\left[\lambda^{z} T, \lambda^{1 / \nu}\left(H-H_{c}\right)\right]+F_{0}(T, H) .
$$

Here $\mathcal{F}(x, y)$ is some a priori unknown function of two variables and $\lambda$ is an arbitrary positive number. This term reflects the singular part of the free energy. We do not even need to make any assumption about the particular value of the exponent $b$ (which is usually set to be $d+z$ - the effective dimensionality of the quantum phase transition). The nonsingular part of free energy $F_{0}(T, H)$ is important at $H \gg H_{c}$ and can be approximated as $-M_{\text {sat }}\left(H-H_{c}\right)$. Then, one can express the magnetization reduction as:

$$
\begin{gathered}
-M_{\mathrm{sat}}+M(T, H)=-\left(\frac{\partial\left(F-F_{0}\right)}{\partial H}\right)_{T} \\
=-\lambda^{b+1 / \nu} \mathcal{M}_{0}\left[\lambda^{z} T, \lambda^{1 / \nu}\left(H_{c}-H\right)\right] .
\end{gathered}
$$

Once again, $\mathcal{M}_{0}(x, y)$ is the unknown function of two variables. Now, at finite temperatures by setting $\lambda=$ $T^{-1 / z}$ one arrives at the following general scaling relation:

$$
1-M(H, T) / M_{\text {sat }}=T^{m} \mathcal{M}\left(\frac{g \mu_{B} \mu_{0}\left(H-H_{c}\right)}{T^{1 / \varphi}}\right) .
$$

Here $\varphi=\nu z$ is the crossover exponent describing the interplay between the thermal and quantum fluctuations in the transition vicinity. The second exponent $m$ also has a simple physical meaning. It describes the temperature dependence of magnetization reduction at $H=H_{c}$.

The exponents $\varphi$ and $m$ are also crucial for characterizing the $T=0$ behavior. To see this, one needs to set $\lambda=\left(H_{c}-H\right)^{-\nu}$ in Eq. (3). Then the zero-temperature magnetization cusp is described as

$$
1-M(H) / M_{\text {sat }} \propto\left(H_{c}-H\right)^{m \varphi} .
$$

So this is the $m \varphi$ product that defines the lowtemperature "cusp singularity", and this is the quantity that needs to be found experimentally in order to verify the "dimensionality reduction" prediction by Jackeli and Zhitomirskii 23 .

Finally, we note that the above discussion yields a generalized version of the "zero scale universality" behavior at $z=2$ critical point in one dimension [24]. The difference is, unlike in the former case neither the numeric values of the corresponding exponents (e.g., $m=1 / 2$, $\varphi=1)$ nor the functional form of $\mathcal{M}(x)$ are predefined.

\section{Quantum critical scaling: Experiment}

The manifestation of the experimentally accessible magnetization quantum critical behavior is contained in
Eq. (4). To verify this relation and determine exponents $\varphi$ and $m$ we studied the $H-T$ scaling of magnetization near saturation in the axial geometry of $\mathrm{BaCdVO}\left(\mathrm{PO}_{4}\right)_{2}$. $M(H, T)$ data measured vs applied field at different temperatures are shown in Fig. 3(a). Equation (4) suggests that all measurements are expected to collapse onto a single curve if rescaled with appropriate exponents. In order to determine the latter, for the data in Fig. 3(a) we defined an empirical goodness of overlap criterion [25]. Overall, this criterion is similar to a standard $\chi^{2}$ with the only difference that the "theoretical" curve with respect to which the deviation of datapoints is calculated is not predefined, but empirically created on the fly at each iteration of the fit. A more detailed description of the algorithm is contained in Appendix A.

Using $\mu_{0} H_{c}=3.95(2) \mathrm{T}$ obtained in calorimetric measurements as described below, we plot $\chi^{2}$ as a function of $m$ and $1 / \varphi$ in the inset in Fig. 3(b). The best overlap is found for $m=0.8(1)$ and $1 / \varphi=1.6(3)$, and results in a spectacular data collapse shown in Fig. 3(b) (main panel). The measured exponents are quite distinct from those in the pure one-dimensional case, where $m=1 / 2$ and $\varphi=1$ [20, 24]. Nonetheless, the observed exponent describing the magnetization cusp in the $T=0$ limit [as given by Eq. (5)] is the same, namely, $m \varphi=0.5 \pm 0.15$, and agrees well with this prediction made for the perfectly frustrated square lattice 23 .

\section{B. Calorimetric studies}

\section{Specific heat measurements}

Further unusual behavior of $\mathrm{BaCdVO}\left(\mathrm{PO}_{4}\right)_{2}$ was revealed by the specific heat measurements. In both the $\mathbf{H} \| \mathbf{a}, \mathbf{b}$ orientations zero-field-cooling data shows a pronounced lambda anomaly at $T_{N}=1.05 \mathrm{~K}$ followed by a power-law decrease in $C_{p}(T) / T$ as shown in Fig. 4 (a). This behavior is fully consistent with the previously reported powder data 12 .

Tracking the phase transition to reconstruct the $H-T$ phase diagram is often easier in constant- $H$ scans, shown in Figs. 4(f)-(j). However, these data reveal a striking difference between axial and transverse geometries. The first key result of our calorimetry studies is that in the axial case, the field-induced transition becomes discontinuous at low temperatures. Above $T^{*} \simeq 0.15 \mathrm{~K}$ both geometries yield a sharp $C_{p}(H)$ peak, marking a secondorder transition [Figs. 4(f)-4(h)] at a critical field $H_{c}$. In the vicinity of $H_{c}$ and at all fields above it the data for the two geometries are virtually indistinguishable. In contrast, below $T^{*}$ the character of the anomaly in the axial geometry changes. As shown in Figs. 4(i) 4(j) it rapidly evolves from a peak to a steplike feature, similar to the step found at the spin flop (a textbook example of discontinuous transition in a magnet).

The second and perhaps the most important finding of our calorimetry experiments is that there is an ad- 

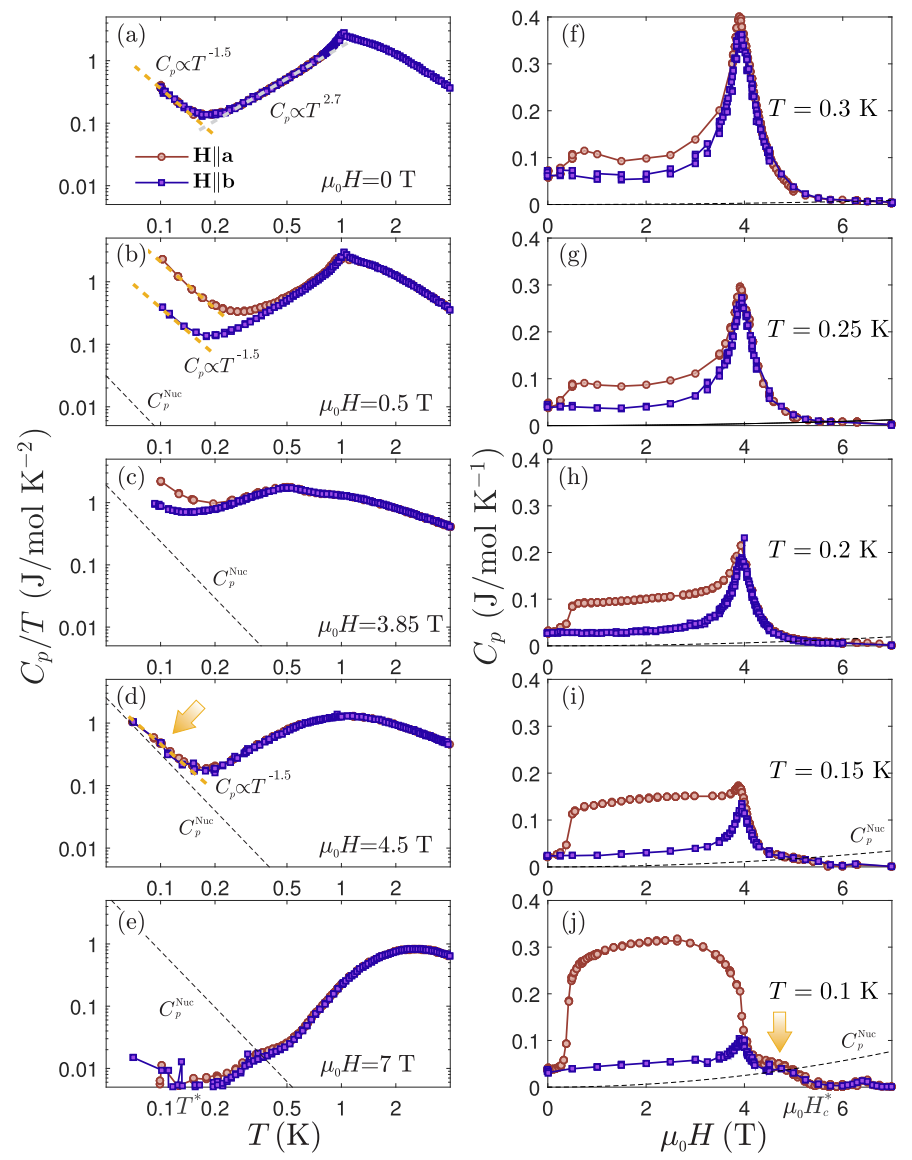

FIG. 4. Low temperature specific heat in $\mathrm{BaCdVO}\left(\mathrm{PO}_{4}\right)_{2}$ for axial and transverse geometries of the magnetic field. (a-e) $C_{p}(H, T) / T$ as the function of $T$ for different magnetic fields. Dashed lines show the power laws that can be identified in the data. (f-j) $C_{p}(H, T)$ at fixed temperature as the function of $H$. Arrows indicate excess specific heat appearing at low temperatures above the field-induced phase transition. In all the plots the dotted lines show the Zeeman effect based estimate of nuclear contribution, Eqs. (677). Please note that the panels on the left have logarithmic scale, while the panels on the right have linear scale. In Appendix B one can also see alternative ways of plotting this data.

ditional anomalous contribution to specific heat at the lowest temperatures above $H_{c}$ in both geometries. It can be seen in both constant- $T$ [Fig. 4(j)] and constant- $H$ scans [Fig. 4(d)]. At $100 \mathrm{mK}$ it persists as a plateau all the way up to $\mu_{0} H_{c}^{*} \simeq 5.2 \mathrm{~T}$, but vanishes at higher fields [Fig. 4(e)].

A very straightforward illustration of vanishing highfield specific heat is shown in Fig. 5 for the axial geometry case. It demonstrates the relaxation curves obtained with the fixed measurement time of $300 \mathrm{~s}$ and magnitude of heating pulse $P=15.8 \mathrm{pW}$ applied for $150 \mathrm{~s}$. The difference between the measurements at fields of $4.5 \mathrm{~T} \gtrsim \mu_{0} H_{c}$ and $8 \mathrm{~T} \gg \mu_{0} H_{c}$ at $T=100 \mathrm{mK}$ is apparent. At $4.5 \mathrm{~T}$ the relaxation curve indeed has the characteristic "shark fin" shape, which means substantial specific heat present

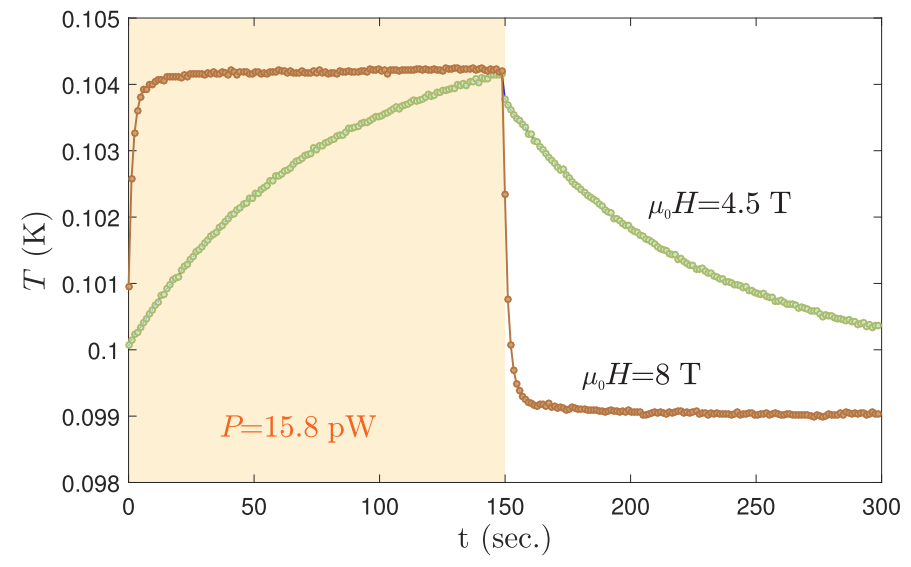

FIG. 5. The raw specific heat data: relaxation curves taken at $\mu_{0} H=4.5$ and $8 \mathrm{~T}$ in axial geometry. The two shown curves correspond to two single datapoints in Fig. 4.j).

(as the rather long measurement period is comparable to the characteristic relaxation time). In contrast, at $8 \mathrm{~T}$ after turning the heater on or off, in a few seconds the system ends up in the stationary regime. This is the clear signature of very short relaxation time and almost absent specific heat in the sample.

\section{Magnetocaloric effect measurements}

The discontinuous character of the low-temperature field-induced transition in the axial case is also confirmed by the measurements of the magnetocaloric effect. Utilizing the same experimental setup as for the relaxation calorimetry, we monitor the sample temperature during slow magnetic field sweeps, while keeping the heat bath temperature constant. In this so-called equilibrium regime [26] the excess thermal power created due to the sample's entropy change is balanced by the temperature gradient between the sample and the bath across the weak heat link. The evolution of the resulting sample's $T(H)$ curves for up and down magnetic field sweeps is shown in Fig. 6. The first-order spin-flop transition manifests itself as a highly asymmetric peaklike feature at all the temperatures. This is a direct consequence of the entropy discontinuity. In contrast, at elevated temperatures the magnetocaloric anomaly at $H_{c}$ is very symmetric, as it should be for a continuous transition [27, 28]. However, below around $T^{*}$ this anomaly rapidly becomes rather asymmetric as well, confirming the change of the transition type.

\section{Possible nuclear specific heat contributions}

Although observations of divergent low-temperature specific heat due to nuclear magnetism with extremely low-energy scale are common, below we will show that 


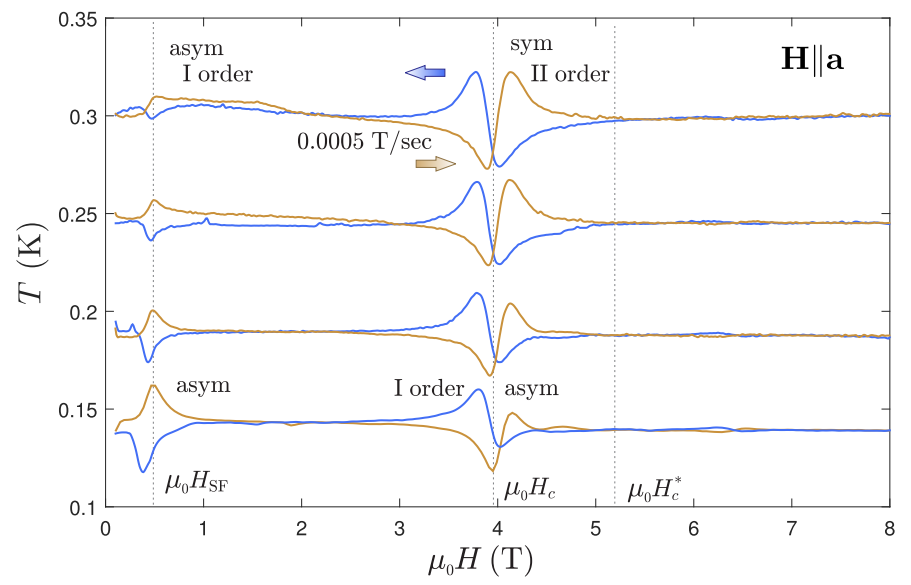

FIG. 6. Magnetocaloric effect in $\mathrm{BaCdVO}\left(\mathrm{PO}_{4}\right)_{2}$ at low temperatures in the axial geometry. The $T(H)$ dependencies taken at different temperatures with the field sweeping rate of $\pm 5 \times 10^{-4} \mathrm{~T} / \mathrm{s}$.

the simple picture is qualitatively inconsistent with the present data.

The simplest model of nuclear specific heat assumes the energy levels in the spinful nuclei being split due to Zeeman effect. Then the nuclear contribution is approximately given as:

$$
C_{p}^{\mathrm{Nuc}}(T, H)=\mathfrak{A}\left(\frac{\mu_{0} H}{T}\right)^{2}
$$

The material-dependent amplitude coefficient is calculated as follows:

$$
\mathfrak{A}=\sum_{i} \mathfrak{A}_{i}=\sum_{i} N_{A} n_{i} \alpha_{i} \frac{I_{i}\left(I_{i}+1\right)\left(\gamma_{i} \hbar\right)^{2}}{3 k_{B}} .
$$

The summation goes through all the spinful types of nuclei present in the material; $n_{i}$ is the stoichiometric coefficient in the chemical formula and $\alpha_{i}$ is the abundance of the particular isotope. The data on the isotopes abundance, nuclear spins $I_{i}$, and corresponding nuclear gyromagnetic ratios $\gamma_{i}$ are found in Ref. [29], for example. The isotope data and the corresponding contribution to the nuclear specific heat prefactor relevant to $\mathrm{BaCdVO}\left(\mathrm{PO}_{4}\right)_{2}$ are summarized in Table II. The overall $C_{p}^{\mathrm{Nuc}}(T, H)$ prefactor is estimated as $\mathfrak{A}=$ $1.5671 \times 10^{-5} \mathrm{~J} \mathrm{~K} / \mathrm{mol} \mathrm{T}^{-2}$, with about $80 \%$ of it stemming from the magnetic ion ${ }^{51} \mathrm{~V}$ having nuclear spin $I=7 / 2$. This means that for the consistent description of $C_{p}^{\text {Nuc }}$ the quadrupolar splitting and hyperfine interactions on the ${ }^{51} \mathrm{~V}$ site also need to be taken into account. These parameters are unknown at the moment, and therefore Eqs. (677) should be seen only as the crude estimate of possible effect magnitude. As one can see from Fig. 4, the low-temperature specific heat in

\begin{tabular}{cccccc}
\hline \hline Isotope & $\alpha_{i}$ & $n_{i}$ & $\gamma_{i}\left(\mathrm{rad} / \mathrm{s} \mathrm{T}^{-1}\right)$ & $I_{i}$ & $\mathfrak{A}_{i}\left(\mathrm{~J} \mathrm{~K} / \mathrm{mol} \mathrm{T}^{-2}\right)$ \\
\hline${ }^{135} \mathrm{Ba}$ & 0.06590 & 1 & $2.6755 \times 10^{7}$ & $3 / 2$ & $2.8604 \times 10^{-8}$ \\
${ }^{137} \mathrm{Ba}$ & 0.11320 & 1 & $2.9930 \times 10^{7}$ & $3 / 2$ & $6.1488 \times 10^{-8}$ \\
${ }^{111} \mathrm{Cd}$ & 0.12750 & 1 & $-5.7046 \times 10^{7}$ & $1 / 2$ & $5.0318 \times 10^{-8}$ \\
${ }^{113} \mathrm{Cd}$ & 0.12260 & 1 & $-5.9609 \times 10^{7}$ & $1 / 2$ & $5.2829 \times 10^{-8}$ \\
${ }^{50} \mathrm{~V}$ & 0.00240 & 1 & $2.6721 \times 10^{7}$ & 6 & $1.1638 \times 10^{-8}$ \\
${ }^{51} \mathrm{~V}$ & 0.99760 & 1 & $7.0492 \times 10^{7}$ & $7 / 2$ & $1.2625 \times 10^{-5}$ \\
${ }^{31} \mathrm{P}$ & 1.00000 & 2 & $1.0839 \times 10^{8}$ & $1 / 2$ & $2.8497 \times 10^{-6}$ \\
${ }^{17} \mathrm{O}$ & 0.00037 & 9 & $-3.6280 \times 10^{7}$ & $5 / 2$ & $6.2013 \times 10^{-9}$ \\
\hline \hline
\end{tabular}

TABLE II. The relevant isotope data (from Ref. [29]) and corresponding calculated contribution [Eq. (7)] to the nuclear specific heat due to Zeeman splitting.

$\mathrm{BaCdVO}\left(\mathrm{PO}_{4}\right)_{2}$ is completely at odds with this simple estimation.

Nonetheless, since nuclear spin $I=7 / 2$ is also carried by the magnetic $S=1 / 2{ }^{51} \mathrm{~V}^{4+}$ ions, some complex behavior induced by hyperfine coupling close to the quantum critical point can not be fully ruled out. There are some experimental [30] and theoretical [31] studies of hyperfine coupled settings, but not for the strongly frustrated $2 \mathrm{D}$ case.

\section{Magnetic phase diagram of $\mathrm{BaCdVO}\left(\mathrm{PO}_{4}\right)_{2}$}

Leaving aside the certainly exotic scenario of interplay between the nuclear and electronic spins, we face the conclusion that the found excess specific heat is of purely electron spin origin. Apart from either electronic or nuclear spins no other degrees of freedom may give a field-dependent contribution to the specific heat of an insulating material at these low temperatures. We conclude that in $\mathrm{BaCdVO}\left(\mathrm{PO}_{4}\right)_{2}$ at the lowest temperatures $H_{c}$ does not correspond to the full saturation. Indeed, the latter would open a Zeeman gap in the spectrum and suppress any magnetic specific heat. Instead, $H_{c}$ indicates the appearance of a new quantum regime with substantial low-energy fluctuations.

The magnetic $H-T$ phase diagram of $\mathrm{BaCdVO}\left(\mathrm{PO}_{4}\right)_{2}$ in Fig.7 7 summarizes the findings. We distinguish conventional paramagnetic (PM), field polarized (FP), and AF states (and its post-spin-flop version $\mathrm{SF}$ ). At intermediate temperatures a quantum critical (QC) regime is observed above $H_{c}$. The new low-temperature field-induced states are labeled as LT. While they are separated from the ordered states by obvious phase transitions, their finite- $T$ boundaries cannot be clearly identified in our calorimetry data. Thus, we simply identify the crossover line below which the anomalous behavior becomes pronounced. The appearance of the LT regime, already intriguing on its own, becomes especially interesting if considered in the context of predictions made for the spin-nematics. 


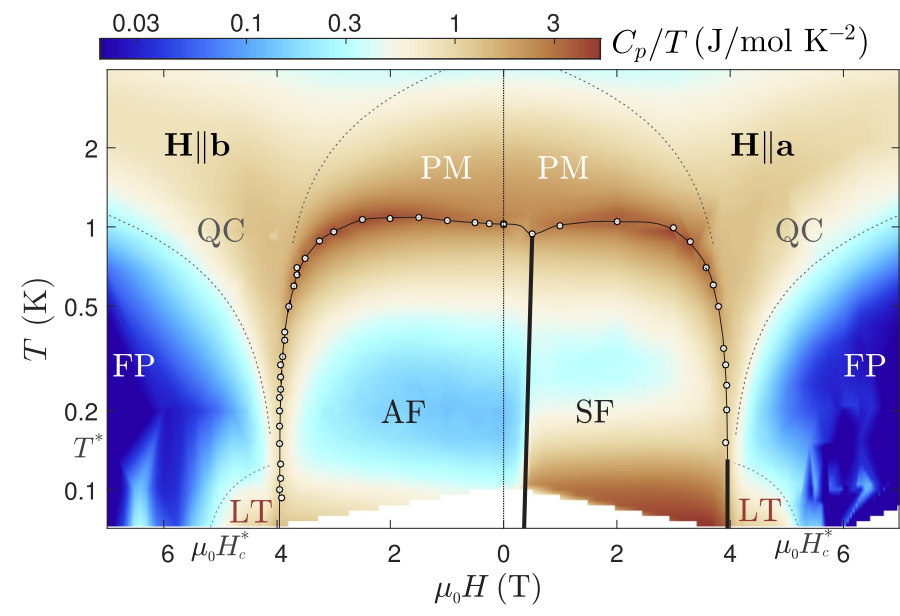

FIG. 7. Magnetic phase diagram for $\mathbf{H} \| \mathbf{a}, \mathbf{b}$. The background shows the false color map of $C_{p}(T, H) / T$, thin and thick black solid lines represent the phase transitions (of second or first order correspondingly), and gray dashed lines mark crossovers. Points are the ordered phase boundary data obtained from $C_{p}$ anomalies. The phases are as follows: PM, paramagnetic; FP, fully polarized; AF, antiferromagnetic; SF, antiferromagnetic after the spin flop; QC, quantum critical regime; LT, unconventional low temperature regime. Crossover lines marking the $\mathrm{QC}$ regime follow $T \propto\left|H-H_{c}\right|^{\varphi}$ with the same crossover exponent $\varphi$ found from scaling Eq. (4).

\section{Observed anomalies in context of spin-nematics}

One can find interesting possible connections of the observed anomalies to the expected behavior of the twodimensional $S=1 / 2$ spin-nematic materials. First, we would like to note that the location of the LT regimes is in principle consistent with the expectations for the spinnematic state in the frustrated square lattice model. The anomalous specific heat found in $\mathrm{BaCdVO}\left(\mathrm{PO}_{4}\right)_{2}$ samples is endemic to the very low temperatures compared to the typical interactions of the order of a few kelvin in the material. Nonetheless, this is indeed the temperature range in which the anomalous behavior due to magnons pairing up is expected to take place from the theory point of view. Exact diagonalization studies of the frustrated model with $J_{2} / J_{1}=-0.4$ suggest significant nematictype contributions to the specific heat to occur at temperatures order of magnitude lower than typical $J$ 's in the system [3]. In the case of $\mathrm{BaCdVO}\left(\mathrm{PO}_{4}\right)_{2}$ the relevant energy scale can be suppressed even further below $0.1 J_{1} \simeq 0.3 \mathrm{~K}$, as the system deviates from the idealized $J_{2} / J_{1}=-0.4$ zero-field case. This looks very consistent with our present observations shown in Fig. 4(d). At the same time the spin-nematic precursor behavior may in principle be present at any field below the true saturation point - as long as there are fluctuating transverse spin components. There are indications that spin fluctuations associated with the new low-temperature high-field phases are present already in the ordered states. There too we find anomalous contributions to specific heat below the crossover temperature $T^{*} \simeq 0.15 \mathrm{~K}$ [Figs. 4(a)4(c)]. They roughly follow $C_{p}(T) \propto T^{-1.5}$ and are particularly strong in the spin-flop state. This suggests their transverse character. The field $H_{c}^{*}$ at which the spin fluctuations vanish is consistent with the effective magnetic energy scale of the material $J_{\text {eff }}=\sqrt{J_{1}^{2}+J_{2}^{2}}$ [7].

A second interesting observation is related to the boundary between conventional antiferromagnetic and LT phases. As shown above, in the axial geometry the new high-field state is entered from the spin-flop AF phase through a discontinuous transition. Incidentally, this is exactly the type of behavior expected for the spin-nematic phase predicted to emerge just below full saturation [16]. The AF and spin-nematic phases have competing order parameters, and therefore the transition between them has to be first order. In the transverse geometry, the spin-nematic phase should not exist in a field due to a lack of axial symmetry 32 . However, this is not supposed to impede the associated fluctuations completely. While strong spin fluctuations persist irrespective of field orientation in $\mathrm{BaCdVO}\left(\mathrm{PO}_{4}\right)_{2}$, they may result in nematic order only in the axial geometry. This may explain why the transition at $H_{c}$ remains continuous in the transverse case and becomes discontinuous in the axial - the only case expected to support the nematic long-range ordering.

To summarize, it is very tempting to consider the observed anomalous regime LT as the precursor of the true spin-nematic long-range order. This would simultaneously explain the small energy scale associated with the new state as well as the field-direction-dependent transition type. However, at this stage we still cannot fully rule out the possibility of interference between the electronic and the nuclear spins going beyond the simple model described by Eq. (7).

\section{CONCLUSIONS}

The high hopes for finding the unconventional magnetism in the frustrated $S=1 / 2$ square lattice magnet $\mathrm{BaCdVO}\left(\mathrm{PO}_{4}\right)_{2}$ appear to be well justified. In addition to the experimentally quantified "dimensionality reduction" effect serving as the indicator of strong frustration we have also found anomalously strong contributions to the specific heat in the vicinity of saturation field at lowest temperatures. Although the possibility of their origin from the interplay of electronic and nuclear magnetism is not yet fully ruled out, these anomalies show qualitative consistency in the order of phase transition and energy scale with the predicted spin-nematic behavior. Future efforts aimed at understanding the origins of the novel regime will have to specifically focus on the lowest possible temperatures. 


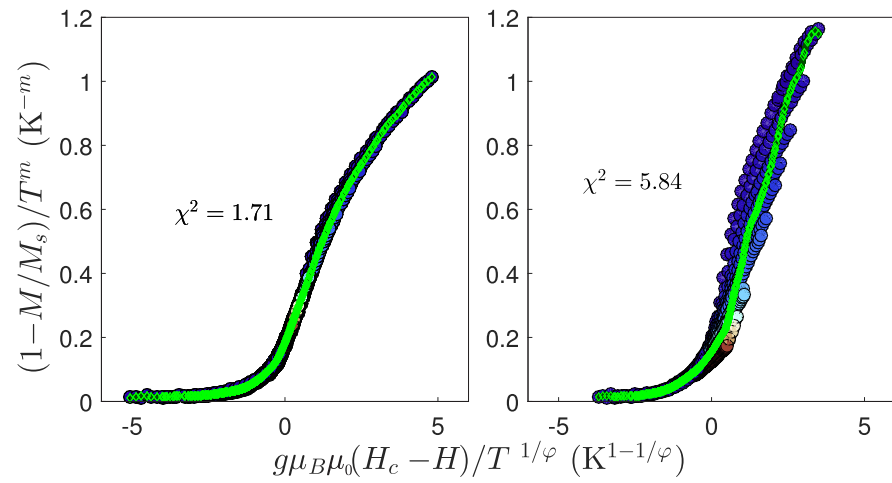

FIG. 8. The scaling analysis of the magnetization data (see Fig. 3 of the main text). Left: optimal scaling exponents $1 / \varphi=1.55, m=0.76$. Right: non-optimal scaling exponents $1 / \varphi=1, m=1$. Green curve represents the empirical data interpolation with respect to which the $\chi^{2}$ costs are calculated.

\section{ACKNOWLEDGMENTS}

This work was supported by Swiss National Science Foundation, Division II. We would like to thank Prof. Oleg Starykh (University of Utah) for enlightening discussions, Stanislaw Galeski and Dominic Blosser (ETH Zürich) for help with the low-temperature magnetocaloric measurements, and Dr. Severian Gvasaliya (ETH Zürich) for technical assistance.

\section{Appendix A: Definition of scaling $\chi^{2}$ criterion}

The hypothesis that is being tested for the magnetization data present in Fig. 3 of the main text is that it follows the universal behavior in the vicinity of $H_{c}$ :

$$
1-M(T, H) / M_{\text {sat }}=T^{m} \mathcal{M}\left(\frac{g \mu_{0} \mu_{B}\left(H_{c}-H\right)}{T^{1 / \varphi}}\right)
$$

This means that for correctly chosen exponents $m$ and $\varphi$ the set of datapoints $X=\frac{g \mu_{0} \mu_{B}\left(H-H_{c}\right)}{T^{1 / \varphi}}$ and $Y=[1-$ $\left.M(H) / M_{\text {sat }}\right] / T^{m}$ should lie close to some hypothetical curve. In principle, if this hypothetical curve $Y_{0}(X)$ is known, the problem of calculating the abstract "goodness of overlap" can be reduced to the very standard problem of calculating of the "goodness of fit" of the data $Y(X)$ by theory $Y_{0}(X)$.

The key idea in the present approach, where no a priori scaling curve is postulated, is to construct $Y_{0}(X)$ "on the fly" based on the current $Y(X)$ data. This is achieved by interpolating the scattered $Y(X)$ with cubic splines. It guarantees the smoothness of the resulting curve and at the same time gives a bit more flexibility than polynomial interpolation used, e.g., in Ref. [20] in a similar situation. The examples of such an empirical interpolation curve for cases with good and poor choices of scaling exponents are shown in Fig. 8 .

A remark needs to be made regarding the normalization of cost function in the case described above. The $\chi^{2}$ value is usually normalized with the number of degrees of freedom, which is typically the number of datapoints. However, in the present situation individual degrees of freedom are rather represented by the individual $M(H)$ scans at fixed temperatures. For any separately taken scan the interpolation procedure would by definition provide an ideal overlap with the "empirical curve", and it is the optimization in the presence of multiple such datasets that constitutes the essence of the procedure. Then the cost function, being the equivalent of a standard normalized error-bar-weighted $\chi^{2}$ is calculated as follows:

$$
\chi^{2}=\frac{1}{N_{\text {Datasets }}-1} \sqrt{\sum_{X_{i}}\left(\frac{Y\left(X_{i}\right)-Y_{0}\left(X_{i}\right)}{\Delta Y\left(X_{i}\right)}\right)^{2}} .
$$

\section{Appendix B: Addenda in the specific heat measurements}

Finally, we would like to present a proof that the observed anomalous specific heat is sample related. One simple consideration is that the addenda contribution is somewhat different in both $\mathbf{H} \| \mathbf{a}$ and $\mathbf{H} \| \mathbf{b}$ cases (as different amounts of grease and a different piece of silver foil holder was used), while the observed extra specific heat is well matched. But even more valuable is the direct comparison, given in Fig. 9 for the $\mathbf{H} \|$ a setup. One can see that the background specific heat contribution is very small. Apart from a tiny Shottky anomaly close to $H=0$ it is dominated by $C_{p} \propto T$ linear specific heat of the silver foil.
[1] A. F. Andreev and I. A. Grishchuk, "Spin nematics," Sov. Phys. JETP 60, 267 (1984).

[2] A. V. Chubukov, "Chiral, nematic, and dimer states in quantum spin chains," Phys. Rev. B 44, 4693 (1991)

[3] N. Shannon, T. Momoi, and P. Sindzingre, "Nematic order in square lattice frustrated ferromagnets," Phys. Rev. Lett. 96, 027213 (2006).
[4] M. E. Zhitomirsky and H. Tsunetsugu, "Magnon pairing in quantum spin nematic," Europhys. Lett. 92, 37001 (2010)

[5] N. Büttgen, K. Nawa, T. Fujita, M. Hagiwara, P. Kuhns, A. Prokofiev, A. P. Reyes, L. E. Svistov, K. Yoshimura, and M. Takigawa, "Search for a spin-nematic phase in the quasi-one-dimensional frustrated magnet $\mathrm{LiCuVO}_{4}$," 

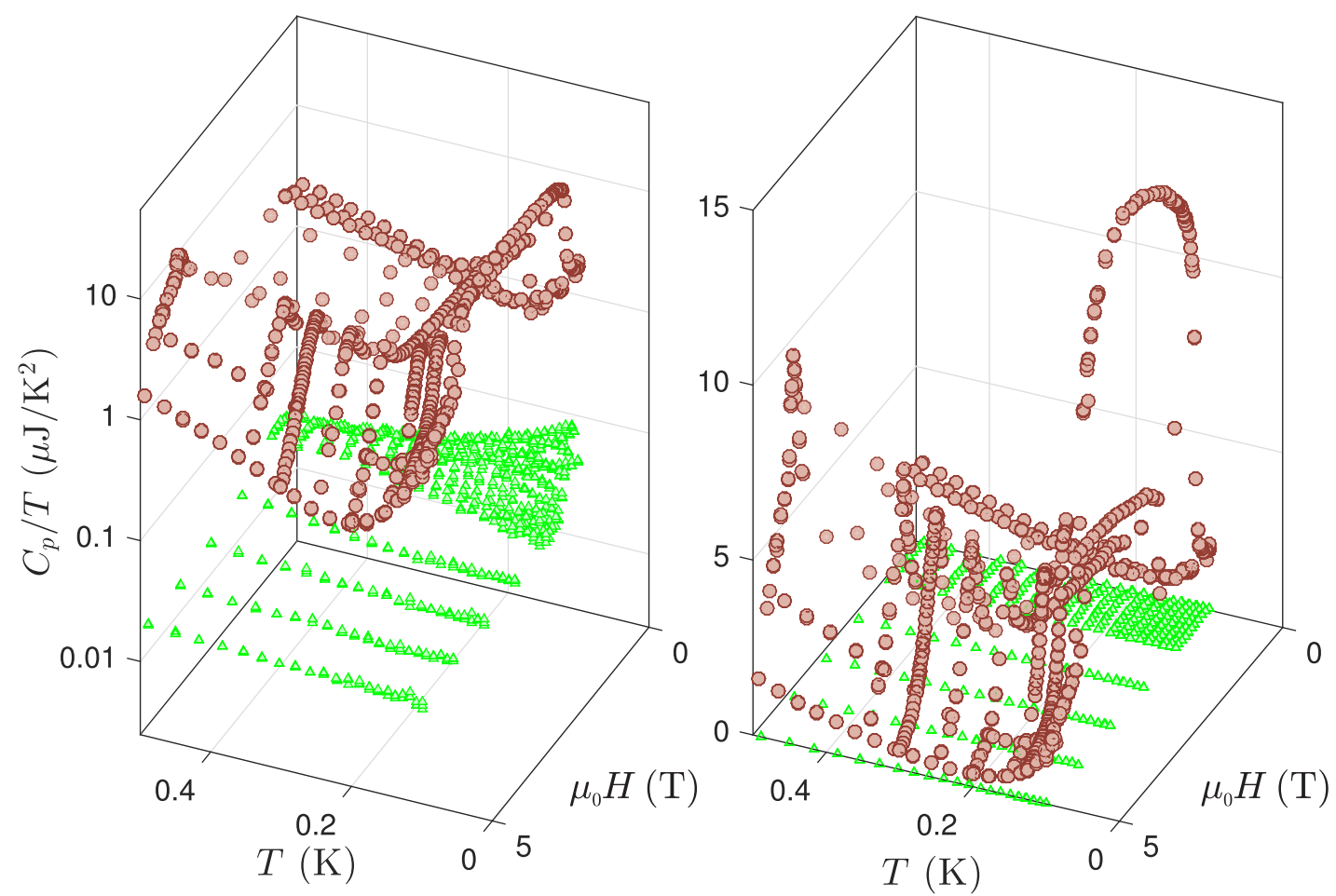

FIG. 9. Low temperature part of addenda specific heat (triangles) and total specific heat (circles) with $\left.\mathrm{BaCdVO}_{(\mathrm{PO}}\right)_{2}$ sample mounted in axial geometry. Left panel shows $C_{p} / T$ data on semilogarithmic scale, right panel shows the data on linear scale.

Phys. Rev. B 90, 134401 (2014)

[6] A. Orlova, E. L. Green, J. M. Law, D. I. Gorbunov, G. Chanda, S. Krämer, M. Horvatić, R. K. Kremer, J. Wosnitza, and G. L. J. A. Rikken, "Nuclear Magnetic Resonance Signature of the Spin-Nematic Phase in $\mathrm{LiCuVO}_{4}$ at High Magnetic Fields," Phys. Rev. Lett. 118, 247201 (2017)

[7] N. Shannon, B. Schmidt, K. Penc, and P. Thalmeier, "Finite temperature properties and frustrated ferromagnetism in a square lattice Heisenberg model," Eur. Phys. J. B 38, 599 (2004)

[8] R. Shindou and T. Momoi, " $S U(2)$ slave-boson formulation of spin nematic states in $S=\frac{1}{2}$ frustrated ferromagnets," Phys. Rev. B 80, 064410 (2009).

[9] R. Shindou, S. Yunoki, and T. Momoi, "Projective studies of spin nematics in a quantum frustrated ferromagnet," Phys. Rev. B 84, 134414 (2011)

[10] B. Schmidt and P. Thalmeier, "Frustrated two dimensional quantum magnets," Phys. Rep. 703, 1 (2017).

[11] H. T. Ueda, "Magnetic Phase Diagram Slightly below the Saturation Field in the Stacked $J_{1}-J_{2}$ Model in the Square Lattice with the $J_{C}$ Interlayer Coupling," J. Phys. Soc. Jpn. 84, 023601 (2015)

[12] R. Nath, A. A. Tsirlin, H. Rosner, and C. Geibel, "Magnetic properties of $\mathrm{BaCdVO}\left(\mathrm{PO}_{4}\right)_{2}$ : A strongly frustrated spin- $\frac{1}{2}$ square lattice close to the quantum critical regime," Phys. Rev. B 78, 064422 (2008)

[13] A. A. Tsirlin, B. Schmidt, Y. Skourski, R. Nath, C. Geibel, and H. Rosner, "Exploring the spin- $\frac{1}{2}$ frustrated square lattice model with high-field magnetization studies," Phys. Rev. B 80, 132407 (2009).
[14] A. A. Tsirlin and H. Rosner, "Extension of the spin- $\frac{1}{2}$ frustrated square lattice model: The case of layered vanadium phosphates," Phys. Rev. B 79, 214417 (2009).

[15] A. Smerald, H. T. Ueda, and N. Shannon, "Theory of inelastic neutron scattering in a field-induced spin-nematic state," Phys. Rev. B 91, 174402 (2015).

[16] A. Smerald and N. Shannon, "Theory of NMR $1 / T_{1}$ relaxation in a quantum spin nematic in an applied magnetic field," Phys. Rev. B 93, 184419 (2016).

[17] S. Meyer, B. Mertens, and Hk. Müller-Buschbaum, "SrZnVO $\left(\mathrm{PO}_{4}\right)_{2}$ and $\mathrm{BaCdVO}\left(\mathrm{PO}_{4}\right)_{2}$ : Vanadylphosphates Related to but not Isotypic with the BaZnVO $\left(\mathrm{PO}_{4}\right)_{2}$ Type." Z. Naturforsch. 52b, 985 (1997)

[18] T. Förster, Elektronenspinresonanz in Systemen mit ferromagnetischen Korrelationen (PhD thesis, TU Dresden, 2011).

[19] P. Thalmeier, M. E. Zhitomirsky, B. Schmidt, and N. Shannon, "Quantum effects in magnetization of $J_{1}-J_{2}$ square lattice antiferromagnet," Phys. Rev. B 77, 104441 (2008)

[20] M. Jeong and H. M. Rønnow, "Quantum critical scaling for a Heisenberg spin- $\frac{1}{2}$ chain around saturation," Phys. Rev. B 92, 180409 (2015).

[21] O. Breunig, M. Garst, A. Klümper, J. Rohrkamp, M. M. Turnbull, and T. Lorenz, "Quantum criticality in the spin-1/2 Heisenberg chain system copper pyrazine dinitrate," Sci. Adv. 3, eaao3773 (2017)

[22] S. E. Sebastian, N. Harrison, C. D. Batista, L. Balicas, M. Jaime, P. A. Sharma, Kawashima N., and I. R. Fisher, "Dimensional reduction at a quantum critical point," Nature 441, 617 (2006). 
[23] G. Jackeli and M. E. Zhitomirsky, "Frustrated Antiferromagnets at High Fields: Bose-Einstein Condensation in Degenerate Spectra," Phys. Rev. Lett. 93, 017201 (2004).

[24] S. Sachdev, T. Senthil, and R. Shankar, "Finitetemperature properties of quantum antiferromagnets in a uniform magnetic field in one and two dimensions," Phys. Rev. B 50, 258 (1994)

[25] K. Yu. Povarov, D. Schmidiger, N. Reynolds, R. Bewley, and A. Zheludev, "Scaling of temporal correlations in an attractive Tomonaga-Luttinger spin liquid," Phys. Rev. B 91, 020406 (2015); M. Hälg, D. Hüvonen, T. Guidi, D. L. Quintero-Castro, M. Boehm, L. P. Regnault, M. Hagiwara, and A. Zheludev, "Finite-temperature scaling of spin correlations in an experimental realization of the one-dimensional Ising quantum critical point," Phys. Rev. B 92, 014412 (2015)

[26] A. A. Aczel, Y. Kohama, C. Marcenat, F. Weickert, M. Jaime, O. E. Ayala-Valenzuela, R. D. McDonald, S. D. Selesnic, H. A. Dabkowska, and G. M. Luke, "FieldInduced Bose-Einstein Condensation of Triplons up to 8
$\mathrm{K}$ in $\mathrm{Sr}_{3} \mathrm{Cr}_{2} \mathrm{O}_{8}$," Phys. Rev. Lett. 103, 207203 (2009).

[27] M. Garst and A. Rosch, "Sign change of the Grüneisen parameter and magnetocaloric effect near quantum critical points," Phys. Rev. B 72, 205129 (2005).

[28] B. Schmidt, P. Thalmeier, and N. Shannon, "Magnetocaloric effect in the frustrated square lattice $J_{1}-J_{2}$ model," Phys. Rev. B 76, 125113 (2007)

[29] J. Mason, Multinuclear NMR (Springer, USA, 1987).

[30] H. M. Rønnow, R. Parthasarathy, J. Jensen, G. Aeppli, T. F. Rosenbaum, and D. F. McMorrow, "Quantum Phase Transition of a Magnet in a Spin Bath," Science 308, 389 (2005)

[31] A. M. Tsvelik and I. A. Zaliznyak, "Heisenberg necklace model in a magnetic field," Phys. Rev. B 94, 075152 (2016)

[32] S.-S. Zhang, N. Kaushal, E. Dagotto, and C. D. Batista, "Spin-orbit interaction driven dimerization in one-dimensional frustrated magnets," Phys. Rev. B 96, 214408 (2017) 